\title{
Capacity Optimization Using Subspace Method Over Multicell OFDMA Networks
}

\author{
Zhu Han, Farrokh R. Farrokhi, Zhu Ji, and K. J. Ray Liu, \\ Department of Electrical and Computer Engineering, University of Maryland, College Park.
}

\begin{abstract}
Capacity optimization in a multi-cell OFDMA system where each cell has multiple users is investigated in this work. The objective is to find an assignment of users to the sets of subcarrier, their transmission rates for the subcarrier, and power allocation such that the total system capacity is increased, while users meet a minimum total rate requirement and a power constraint. Since the optimal solution involves an exhaustive search or complex nonlinear integer programming, we develop sub-optimal low complexity algorithms. We propose a two-step scheme: First an initial channel and data rate allocations are determined by two initialization algorithms. Then we refine the assigned rates by an iterative algorithm. From simulation results, the proposed algorithms can efficiently allocate resources to increase the overall system capacity and reduce the allocation outages.
\end{abstract}

\section{INTRODUCTION}

In multiuser wireless systems, users to users channel variations, due to location differences and fading in time and frequency, can be utilized to improve system capacity. By assigning bandwidth according to users' channel responses, spectral efficiencies can be improved. This technique, which is known as multiuser diversity, in Orthogonal Frequency Domain Multi-Access (OFDMA) can be utilized over time and frequency. However, in order to maintain the basic link qualities, the allocation algorithm has to efficiently utilize the bandwidth to increase system capacity and at the same time meet the minimal data rate requirements of different users.

This problem has been of interest recently. In [1], [2], in single cell systems, suboptimal algorithms were proposed such that total transmit power was minimized and a minimum rate requirement for each user was to be satisfied. In [3], a suboptimal simple algorithm was proposed for single cell case. In [4], a similar problem in a single cell system was formulated as max-min user throughput optimization under a maximum transmit power policy. In [5], the objective was defined as maximizing total system throughput, in a multi-cell system, while the transmit power per user was limited. In that work, a suboptimal water-pouring based algorithm was proposed to solve that problem. A number of heuristic algorithms were proposed in [6] to find feasible channel assignments and transmit power allocation in multi-cell systems. Most of the previous works concentrate on either single cell channel assignment problem or multicell power control problem. Very few works address the multi-cell OFDMA resource allocation where each cell has multiple users, which is a very difficult high dimension assignment and nonlinear problem. This motivates us to study this problem and try to find a possible solution.

In this work the problem of capacity optimization by dynamic allocation of subcarrier to users in a multi-cell OFDMA network is investigated. The objective is defined as to maximize the overall system capacity while a minimum rate requirement for each user can be satisfied and the transmitted power is constrained. Since this problem is NP hard, we propose a two-step suboptimal scheme which can serve as a benchmark. In the initialization step, we develop two algorithms: First, we start from an equal rate channel allocation across users; in another approach, we start from a maximum packing solution. In the refinement step, we improve system capacity by an iterative algorithm. Through the numerical studies, we will show that the proposed algorithms can efficiently allocate the resource to increase the system capacity and reduce the outage probability, when the system is more crowed.

In Section II, we present the system model and problem definition. In Section III, we propose two initialization algorithms. In Section IV, we develop an iterative capacity refinement algorithm by using subspace methods. Numerical studies are included in Section V. Finally, in Section VI, the contribution of this work is summarized.

\section{System Model And Problem Definition}

Assume there are $N$ cells in the system and the $i^{\text {th }}$ cell has $M_{i}$ mobile users. There are totaly $K$ subcarrier in the system. Within each cell, only one user is allocated to each subcarrier. Among different cells, multiple users share the same subcarrier. The allocations of users and powers to subcarrier are denoted by $K \times N$ matrices $\mathbf{A}$ and $\mathbf{P}$, respectively. $[\mathbf{A}]_{k i}=A_{k i}$ represents user number $j$ that occupies the $k^{t h}$ subcarrier in the $i^{t h}$ cell. $A_{k i} \in\left[1, \ldots, M_{i}\right] .[\mathbf{P}]_{k i}$ is this user's power. For the uplink case, in the $i^{\text {th }}$ cell, the $j^{\text {th }}$ user occupies the $k^{t h}$ subcarrier, i.e., $A_{k i}=j$. We assume the system is synchronized and our results can be served as a benchmark for the distributed unsynchronized system. The uplink received SINR is:

$$
\Gamma_{i}^{k}=\frac{P_{i}^{k} G_{i i}^{k}}{\sum_{l \neq i} P_{l}^{k} G_{l i}^{k}+N_{0}}
$$

where $P_{i}^{k}$ is the transmit power from $i^{t h}$ cell for the $k^{t h}$ subcarrier, $G_{l i}^{k}$ is the interferer's propagation loss from the $l^{t h}$ cell to the $i^{t h}$ cell for the $k^{t h}$ subcarrier, and $N_{0}$ is the sampled thermal noise level. Without loss of generality, we 
assume the noise level is the same for all users. Suppose a target SINR $\gamma_{i}\left(\Gamma_{i} \geq \gamma_{i}\right)$, in matrix form, we have

$$
\left(I-\mathbf{D}^{k} \mathbf{F}^{k}\right) \mathbf{P}^{k}=\mathbf{u}^{k}
$$

where $\mathbf{P}^{k}=\left[P_{1}^{k}, \ldots, P_{N}^{k}\right]^{T}, \mathbf{D}^{k}=\operatorname{diag}\left(\gamma_{1}^{k}, \ldots, \gamma_{N}^{k}\right), \mathbf{u}^{k}=$ $\left[u_{1}^{k}, \ldots, u_{N}^{k}\right]^{T}, u_{i}^{k}=\gamma_{i} N_{0} / G_{i i}$, and

$$
\left[\mathbf{F}_{i j}^{k}\right]= \begin{cases}0 & \text { if } j=i \\ \frac{G_{j i}}{G_{i i}} & \text { if } j \neq i .\end{cases}
$$

The above equation has a solution with possible power vector, if the spectral radius (the maximal eigenvalue) of $\rho\left(\mathbf{D}^{k} \mathbf{F}^{k}\right)$ is inside unit circle [8].

We assume that the channels change slowly and are stable over a frame with hundreds of symbols. Assume $A_{k i}=j$, the capacity is denoted by

$$
c_{i j}^{k}=W \log \left(1+\frac{\Gamma_{i}^{k}}{\Gamma}\right),
$$

where $\Gamma$ is a constant for capacity gap and $W$ is the bandwidth. Without loss of generality, we assume $W=1$.

The goal of this paper is to maximize the system overall capacity. Each user has a minimal rate requirement $R_{i j}$ when he is admitted to the system. In practice, the transmitted power of each user is bounded by $P_{\max }$. The users will water fill their powers to the carefully assigned channels according to the channel responses, interferences, and noises. This will involve complicated channel assignment and high dimension nonlinear optimization. In [9], it has been shown that the power is closely related to the spectral radius of $\mathbf{D}^{k} \mathbf{F}^{k}$. In this paper, to simplify the problem, we let the spectral radius to be bound by $1-\epsilon$, where $\epsilon$ is a small number. We can carefully select its value, such that the power constraint is satisfied. The constrained optimization problem can be expressed as:

$$
\begin{gathered}
\max _{\mathbf{A}, \mathbf{P}} \sum_{i=1}^{N} \sum_{j=1}^{M_{i}} \sum_{k=1}^{K} c_{i j}^{k} \\
\text { s.t. } \begin{cases}\text { Rate: } \quad & c_{i j}=\sum_{k=1}^{K} c_{i j}^{k} \geq R_{i j}, \forall i, j . \\
\text { Power: } \quad\left|\rho\left(\mathbf{D}^{k} \mathbf{F}^{k}\right)\right| \leq 1-\epsilon, \forall k .\end{cases}
\end{gathered}
$$

Since finding the optimal solution to the problem in (5) directly is extremely complicated and may involve complicated nonlinear large dimension integer programming or even exhaustive search. For example, by using Monte Carlo method with multiple initializations or simulation annealing, we can achieve some local optima or even global optimum. However the complexity is too large even for performance analysis. So we try to solve it in two steps to reduce the complexity. In the first step, we initialize the resource allocation by fast suboptimal algorithms to allocate channels and powers. In the second step of refinement, for each subcarrier, we develop an iterative algorithm to increase the system capacity subject to the minimal rate and power constraints per user.
TABLE I

Initialization ALgOrithm A

1. For each subcarrier $k$ from the pool of available subcarrier, search over the possible system to maximize the spectral radius of the matrix $\mathbf{F}^{k}$, i.e., $\gamma^{k}=\max _{\mathbf{A}}(1-\epsilon) / \rho\left(\mathbf{F}^{k}\right)$.

2. In the set of available subcarrier, start from the subcarrier with the best SINR and assign equal rates (a function of SINR) to assigned users in that carrier, and remove the subcarrier from the search.

3. Remove users that achieve the desired rate from the search.

4. Continue with a new subcarrier in Step 1, until all users have the minimal rate requirement.

5. Allocate the rest of subcarrier in a greedy way.

\section{Initialization Algorithms}

In this section, we present two algorithms for initializing resource allocations. In the first algorithm, we find a channel assignment that maximizes the equally achieved rate for users. In the second approach, we pack an initial set of users plus their channel and rate assignments such that total system capacity is optimized. Using any of the allocation schemes, we enhance the system capacity by an iterative algorithm in the next section.

\section{A. Equal SINR/Rate Allocation}

In the first algorithm, we consider a system where each base station allocates one user to each subcarrier. The objective for user assignment is to select one user from each cell for each subcarrier and form the best set of users that maximizes capacity. For the case of equal SINR allocation to all users, this problem is equivalent to finding the best allocation of users that minimizes the spectral radius of the gain matrix, $\mathbf{D}^{k} \mathbf{F}^{k}$, for each carrier, i.e.,

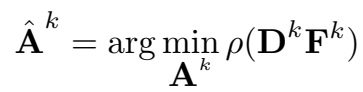

where $\mathbf{A}^{k}$ is the $k^{\text {th }}$ column of $\mathbf{A}$ which consists of the indices of users allocated in different cells for the $k^{t h}$ subcarrier.

The optimal solution finds the best user for each subcarrier, the maximum equal SINR for each allocation, and the power allocation to achieve the maximum SINR. The power allocation can be calculated from (2). The difficulty is to find the best user assignment for each subcarrier, which involves an exhaustive search over all users. Here we present a suboptimal approach to find the user allocation. We find the user that minimizes the link gain for each subcarrier, i.e.,

$$
\hat{A}_{k i}=\arg \min _{A_{k i}} 1 / G_{i i}^{k}
$$


After the above user allocation, we find the maximal achievable $\gamma^{k}$ for this subcarrier. Then we try to find the best allocation for the next subcarrier. If a user's minimum rate requirement is satisfied, this user is excluded from further resource allocation, until all the users have their minimal rate requirements. Finally, the rest of the subcarrier is greedily allocated to the users with the best channel conditions. The algorithm is shown in Table I, where $\epsilon$ is a small number. The maximal transmitted power $P_{\max }$ will determine its value and $\rho \leq 1-\epsilon$. This algorithm can be implemented in a distributive manner with limited communications between base stations.

\section{B. Maximal Rate Packing}

In the second algorithm, we find the best set of users for each subcarrier and each subcarrier is not necessarily occupied by all base stations. The basic idea is to pack each subcarrier with the best users in the networks as long as the capacity is increasing.

First, the algorithm finds the highest SINR user and subcarrier in the networks. This user maximizes the channel capacity for this subcarrier. Then we add users one by one to share the subcarrier. If adding users does not improve the total capacity for this subcarrier, the assignment is stopped and we continue for the remaining subcarrier. If any user is allocated more than its desired rate, he will be removed from the future optimization list. The algorithm continues until all users have the minimal rate requirements. When the algorithm is not able to find a solution, due to lack of resources, we report an outage. Otherwise, the rest of the subcarrier is assigned by a greedy method where we pack the subcarrier with the same method above, but users are not removed from the list, such that the total network capacity is increased.

In this approach, the maximal data rate is packed in the network for each subcarrier independent of their cells. That means some base stations may or may not assign a specific subcarrier to their users. In using a subcarrier, the base station sacrifices for the other cells with the hope that the other base stations will run out of users and reduce interferences in other subcarrier. The algorithm is shown in Table II. To implement this algorithm, we need a centralized control and sufficient channel estimations. So the algorithm fits the situation where the number of cells is small and channel changes slowly.

\section{Capacity Refinement Algorithm}

We have presented initialization algorithms for the channel and power allocations for different subcarrier for different users in different cells. In this section, we will develop a two-step iterative algorithm to refine the allocation such that the system overall capacity can be improved under the rate and power constraints. In the first step, we improve the system feasibility. We find the gradient $\partial \rho\left(\mathbf{D}^{k} \mathbf{F}^{k}\right) / \partial \Gamma_{i}^{k}$ for the $k^{t h}$ subcarrier and then project this gradient onto the
TABLE II

Initialization Algorithm B

1. Evaluate maximum data rate for each user at its maximum transmission power for each subcarrier. Start with the subcarrier and user with the highest rate

2. Add one user at a time and find the best user and assigned power that maximizes the total capacity.

3. Repeat Step 2 until total capacity does not increase by adding users.

4. Repeat from first step with a new subcarrier, until all users satisfy their rate requirement.

If no solution, report an outage.

5. Allocate the rest of subcarrier in a greedy way.

plane where the overall capacity for this subcarrier is fixed. Then we move along this modified gradient so that $\rho\left(\mathbf{D}^{k} \mathbf{F}^{k}\right)$ is reduced, while the overall capacity of this subcarrier is maintained the same. In the second step, we increase each subcarrier's SINR for different users to increase the system performance until the system is almost infeasible. Here we consider the users whose rates are less than $R_{i j}$ first, because their rates may be impaired by the first step. The two steps are executed iteratively to improve the system capacity. The iteration stops when reaching the boundary or some stable point.

In the first step, we find the gradient first. It has been shown that the existence of the derivative of the spectral radius $\rho\left(\mathbf{D}^{k} \mathbf{F}^{k}\right)$ by the following theorem [7].

Theorem 1: let $\lambda$ be a simple eigenvalue of $\mathbf{D F}$, with right and left eigenvectors $\mathbf{x}$ and $\mathbf{y}$, respectively. let $\tilde{\mathbf{F}}=\mathbf{D F}+\mathbf{E}$, where $\mathbf{E}$ is a small perturbation. There exists a unique $\tilde{\lambda}$, eigenvalue of $\tilde{F}$ such that

$$
\tilde{\lambda}=\lambda+\frac{\mathbf{y}^{H} \mathbf{E x}}{\mathbf{y}^{H} \mathbf{x}}+\mathrm{O}\left(\|\mathbf{E}\|^{2}\right)
$$

Proof: [8]

In our application, we only try to reduce the maximum absolute eigenvalue. Let $\mathbf{x}^{\mathbf{k}}$ and $\mathbf{y}^{\mathbf{k}}$ be the eigenvectors of the largest eigenvalue. Define $\mathbf{E}^{k}=\Delta \Gamma_{i}^{k} \mathbf{F}_{i}^{k}$, where

$$
\left(\mathbf{F}_{i}^{k}\right)_{j l}=\left\{\begin{array}{cc}
0, & j \neq i \\
\left(\mathbf{F}^{k}\right)_{j l}, & j=i .
\end{array}\right.
$$

We can have the gradient to reduce spectral radius as:

$$
g_{i}^{k}=\frac{\partial \rho\left(\mathbf{D}^{k} \mathbf{F}^{k}\right)}{\partial \Gamma_{i}^{k}}=\frac{\left(\mathbf{y}^{k}\right)^{H} \mathbf{F}_{i}^{k} \mathbf{x}^{k}}{\left(\mathbf{y}^{k}\right)^{H} \mathbf{x}^{k}} .
$$

If we change each user's SINR, according to above gradient $\mathbf{g}^{k}=\left[g_{1}^{k} \ldots g_{N}^{k}\right]^{T}$, the capacity of each subcarrier will be reduced. In [9], we project the gradient to a plane where the overall capacity is a constant. The plane, that is tangent to 
the curve where overall capacity is equal, can be expressed as:

$$
\sum_{i=1}^{N} b_{i} x_{i}=C
$$

where $b_{i}^{k}=1 /\left(1+\Gamma_{i}^{k}\right)$ and $C$ is a constant. The projected gradient is $\mathbf{h}^{k}$, i.e the gradients in (10) projected onto the plane in (11). However if we move along this gradient, some users' rates may be reduced below the minimal rate requirement. We will compensate back the rates in the second step. The first step is stopped when the resource allocation falls to a local optimum or hits the boundary, i.e., some user' SINR for some subcarrier is reduced to zero.

In the second step of the iterative algorithm, we will optimize the overall system capacity. The gradient of overall capacity with respect to targeted SINR is given by:

$$
q_{i}^{k}=\frac{\partial c_{i}^{k}}{\partial \rho\left(\mathbf{D}^{k} \mathbf{F}^{k}\right)}=\frac{\partial c_{i}^{k}}{\partial \Gamma_{i}^{k}} / \frac{\partial \rho\left(\mathbf{D}^{k} \mathbf{F}^{k}\right)}{\partial \Gamma_{i}^{k}}=\frac{1}{g_{i}^{k}\left(\Gamma+\Gamma_{i}^{k}\right)}
$$

We will change the SINRs of the users whose rates are below the minimal requirements first, while keeping other users' SINR fixed, until all users' requirements are satisfied. Then we increase SINR of all users' according to this gradient to increase the overall system capacity, until we hit the boundary, i.e. $\rho\left(\mathbf{D}^{k} \mathbf{F}^{k}\right)=1-\epsilon$.

We repeat the above two steps until the results are stable. We observe that the second step may stop when the constraints are not satisfied. If the results satisfy the minimal rate constraint, we will return these results, otherwise we will return the results in the previous iteration. Then the channel and power allocation is selected for different users. The whole algorithm is operated within the feasible region and the solution is on the boundaries. Define $\mu$ and $\mu^{\prime}$ as small constants, their values determines the converges speed and the accuracy of the final results. Since the algorithm is initialized with a feasible solution and users' targeted SINRs are modified within the feasible range, the proposed algorithm always converges. The iterative capacity Improvement algorithm is given in Table III.

Similar to initialization algorithm B, the improvement algorithm needs a centralized control and many channel estimations. So it only fits small scale systems. Moreover, users' minimal rate may be reduced in the first step of the improvement algorithm and cannot be compensated back in the second step. Under this condition, there are outages when the minimal rate requirement is not satisfied, and we just simply switch back to the original settings determined by the initialization algorithms.

\section{Simulations}

In order to evaluate the performances of the proposed algorithms, a network with $N=7$ is simulated in Fig. 1. One base station is located as the center of each cell. Each cell has the same number of users $M_{i}=3, \forall i$ and all the users are randomly located within each cell. The total number
TABLE III

Iterative CAPacity Improvement Algorithm
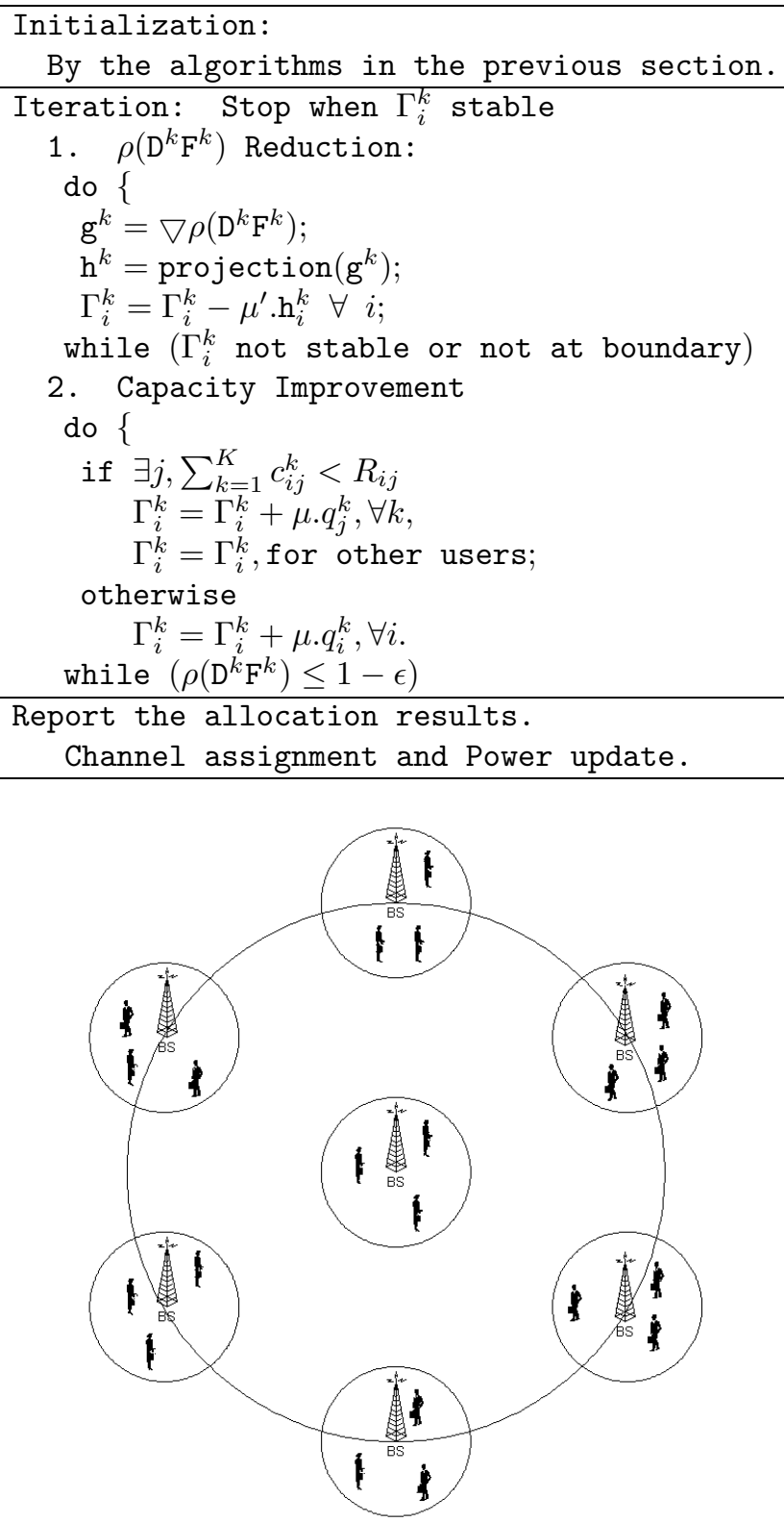

Fig. 1. Simulation Setup

of subcarrier is $K=32$ and each subcarrier is assumed to have unit bandwidth. Each cell's radius is $200 \mathrm{~m}$. The distance between base stations over the cell radius is 3 . The maximal power is $P_{\max }=0.5$ Watts. $\Gamma=1$. Each user has the same thermal noise level $-80 \mathrm{dBm}$. The propagation loss factor is 3.5. The maximal doppler frequency shift is $100 \mathrm{~Hz}$ and four-path frequency selective Rayleigh fading channels are simulated, which has an exponential power profile with 100ns root-mean-square (RMS) delay spread.

In Fig. 2, we show the average power per user vs. spectral radius. We show the result of algorithm $\mathrm{A}$ and improvement algorithm for algorithm A. We can find that even though 


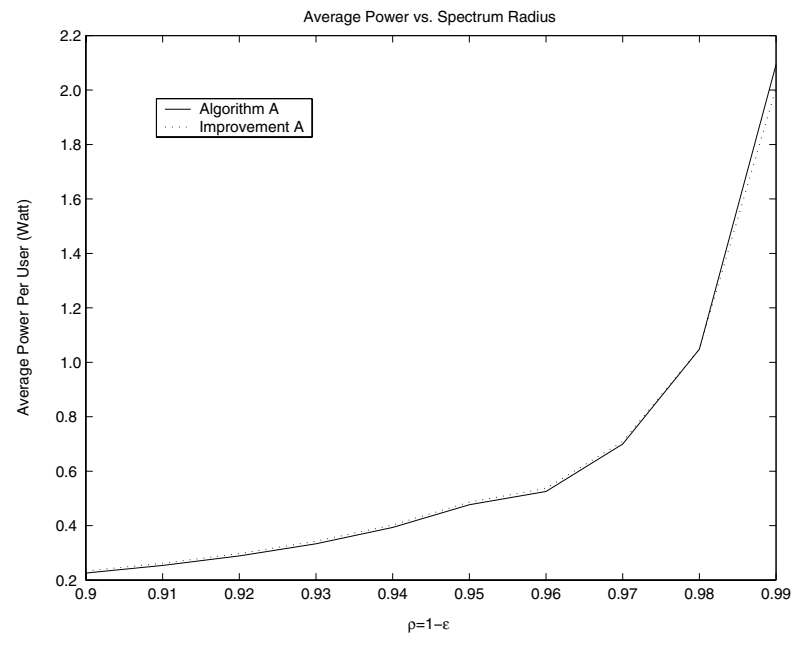

Fig. 2. Average Power vs. Spectral Radius $\rho$

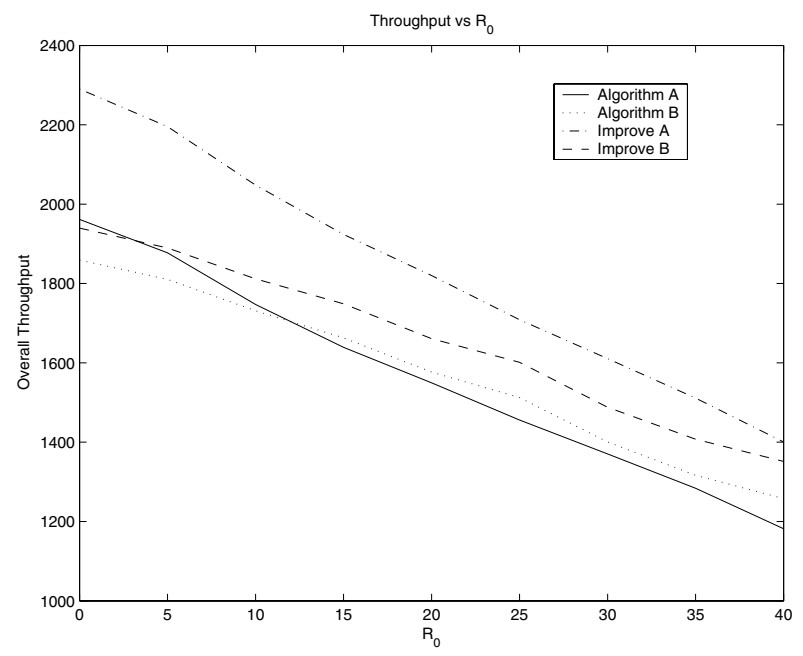

Fig. 3. Overall Capacity vs. Minimal Rate $R_{0}$

the rate and power allocations are quite different, for the same spectral radius constraint, the average powers of two schemes are almost the same. This means that it is reasonable to replace the power constraint by the spectral radius constraint in the problem formulation in (5). The powers increase fast when the spectral radius approaches 1 . We select $\rho=0.95$ in our simulations such that the power constraint is limited to less than 0.5Watts.

In Fig. 3, we show the overall system capacity vs. the minimal rate requirement $R_{0}$ for each user for algorithm A, algorithm B, improved algorithm A, and improved algorithm B. We can see that the overall capacity is reduce when $R_{0}$ is increasing for all algorithms. This is because the system is more fair and has to give more resources to the users with bad channels. Algorithm B has slightly better performance than algorithm $\mathrm{A}$ when $R_{0}$ is large. This is because each subcarrier is optimally occupied by users. Algorithm A has a little bit better performance than algo-

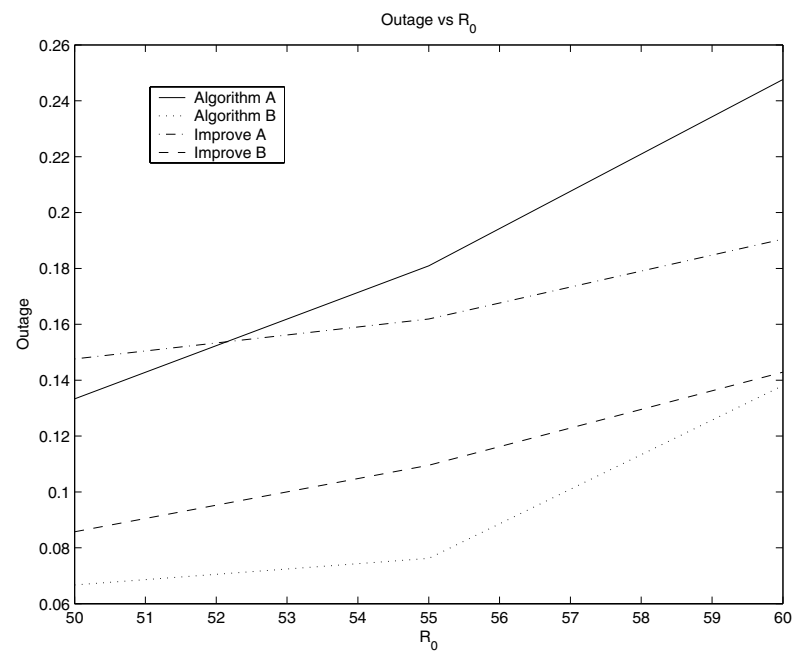

Fig. 4. Outage vs. Minimal Rate $R_{0}$

rithm $\mathrm{B}$ when $R_{0}$ is small. This is because when algorithm B satisfies most of users' minimal rate, the last few users will waste the resources because there are no other users that can share the subcarrier, while a large number of users get the minimal rate at the same time by the equal SINR algorithm A. The improvement algorithm can improve the performance of both algorithm $\mathrm{A}$ and algorithm $\mathrm{B}$, while the improvement for algorithm $\mathrm{A}$ is much larger than that for algorithm B. This is because each carrier is occupied by much more users for algorithm A than for algorithm B. Consequently, the improvement algorithm can have much more room to reduce spectral radius and increase the overall capacity iteratively.

In Fig. 4, we show the outage percentage (the ratio of the number of users that cannot be satisfied with the minimal rate over the total number of users) vs. $R_{0}$. We can see that the outage percentage increases when $R_{0}$ is increasing. Algorithm B has much lower outage rate than algorithm A. This is because algorithm B can pack more rate for each subcarrier. Improvement algorithm can reduce the outage rate when $R_{0}$ is large. But when $R_{0}$ is not large enough, the improvement algorithm has higher outage rate. Under this condition, we will switch back to the original solution of the initialization algorithms.

\section{Conclusions}

In this paper, we study how to increase the capacity for multi-cell OFDMA systems where each cell has multiple users. The difficulties are the channel assignment within each cell and power control among cells. The goal is to develop a less complex scheme to optimize the system capacity under the constraint of minimal rate and power constraints for each user by adaptive channel assignment and power allocations. We develop two algorithms for initial resource allocation and one iterative algorithm to improve the performance. From the simulation results, the proposed algo- 
rithms can provide a good performance benchmark for this complicated resource allocation problem.

\section{REFERENCES}

[1] C.W. Wong, R.S. Cheng, K.B. Lataief, and R.D. Murch, "Multiuser OFDM with apdative subcarrier, bit and power allocation", IEEE JSAC, 1999.

[2] S. Pietrzyk and G.J.M Janssen, "Multiuser subcarrier allocation for QoS provisioning in the OFDMA systems", IEEE Vehicular Technology Conference, Vancouver, Canada, Sep. 2002.

[3] H. Yin and H. Liu, "An efficient multiuser loading algorithm for OFDM-based broadband wireless systems," IEEE Global Telecomm. Conference, vol.1, pp. 103-107, 2000.

[4] W. Yu, W. Rhee, S. Boyd, and J.M. Cioffi, "Iterative water-filling for gaussian vector multiple access channels", to appear in IEEE Transactions on Information Theory.

[5] H. J. Su and E. Geraniotis, "A distributed power allocation algorithm with adaptive modulation for multi-cell OFDM sytems," 5 th IEEE International Symposium on Spread Spectrum Techniques and Applications, vol. 2, pp. 474-478, 1998.

[6] I. Koutsopoulos and L. Tassiulas, "Channel state-adaptive techniques for throughput enhancement in wireless broadband networks," Proceedings of IEEE INFOCOM, pp. 757-766, 2001.

[7] A. Mercado and K.J. R. Liu, "NP-hardness of the stable matrix in unit interval family problem in discrete time", Systems and Control Letters, pp. 261-265, April 2001.

[8] G. Golub, "Matrix computations, third edition", Johns Hopkins University Press, 1996.

[9] Z. Han and K.J. Ray Liu, "Throughput maximization using adaptive modulation in wireless networks with fairness constraint", IEEE WCNCO3, 2003. 